\title{
Optical Link Design for Minimum Power Consumption and Maximum Capacity
}

\author{
N.J. Doran and A D Ellis \\ Aston Institute of Photonic Technologies, Aston University, Birmingham B4 7ET, UK. \\ n.j.doran@aston.ac.uk
}

\begin{abstract}
Using a well-established analytic nonlinear signal-to-noise ratio noise model we show that there are very simple, fibre independent, amplifier gains which minimize the total energy requirement for amplified systems. Power savings of over $50 \%$ are shown to be possible by choosing appropriate amplifier gain and output power.
\end{abstract}

\section{Introduction}

Optical links are generally designed to carry the maximum data capacity for the longest distance at lowest cost. Key design parameters available to planners are the signal powers and amplifier spacing. The specific performance achievable will in addition depend on parameters such as fibre loss, nonlinear coefficient and modulation formats. Fibre nonlinearity limits the Shannon limited capacity and simple models of this ultimate limit have recently been derived [1, 2]. Advances in modulation format and FEC have allowed this limit to be approached in practical systems [3].

The optimum signal powers which allow the nonlinear limit for capacity to be reached are key results of these works. But all this previous work sought to maximise the system performance and optimised the signal power only in respect to performance. Contrastingly, here we shall investigate the total optical power required for an entire system and identify the amplifier spacing which minimises this power allowing systems to be designed with the lowest energy requirements. This will be important both from a simple operating cost angle as well as providing a significant contribution to reducing energy in communication systems across the globe. In particular we will show that there is a very simple prescription for the optimum amplifier gain (and thus span length) to obtain the best power performance overall.

This is also the lowest energy state for the system and our results demonstrate the available energy savings over conventional designs (over 50\%) and should give engineers a target for minimising future energy demand.

\section{General analytical results}

For a coherently detected optical link of $\mathrm{N}$ amplifiers with equal gain the nonlinear impact on the signal-to-noise ratio (SNR) can be expressed as a simple additional noise term, cubic in signal power This SNR in turn can be used to derive the channel capacity limit, or the performance of a specific modulation format by substitution into the conventional equations. In this paper we consider a specific formalism [1] but the results may be generalised to all current calculations of nonlinear capacity [2].

$$
S N R=\frac{P_{S}}{N(G-1) \sigma_{A S E}+N L_{e f f} \sigma_{N L} P_{S}^{3}}
$$

Where $P_{S}$ is the channel power, $G$ the amplifier gain, $L_{\text {eff }}$ the nonlinear effective length of each span and $\sigma_{A S E}, \sigma_{N L}$ are considered length independent parameters deteriming the strength of the amplified spontaneous and nonlinear noise respectively and are given by

$$
\sigma_{\text {ASE }}=n_{s p} h v, \sigma_{N L}=\frac{2 \pi \gamma^{2} c}{\pi \lambda^{2} D} \log \left(\frac{\pi B_{a m p} \lambda^{2} D}{\alpha c}\right)
$$

where $B_{\text {amp }}$ represents the overall bandwidth of the system and the remaining parameters have their usual meanings. Equation 1 has a very simple optimum which occurs when the nonlinear term $=1 / 2 P_{A S E}$. A simple analytic form for this optimum power has been derived $[1,2]$..

$$
P_{0}=\sqrt[3]{\frac{\alpha G \sigma_{A S E}}{2 \sigma_{N L}}}
$$

In order to investigate the total power used in a system consisting of $\mathrm{N}$ amplifiers we now rewrite equations 1 and 2 in terms of the total power $P_{T}$ (at fixed span length $L$ )

$$
\begin{gathered}
S N R_{T}=\frac{N P_{T}}{N^{3}(G-1) \sigma_{A S E}+L_{e f f} \sigma_{N L} P_{T}^{3}} \\
P_{T}=N P_{0}
\end{gathered}
$$

We can consider two approaches to calculating the minimum total signal power. The first is to take the optical power given by equation 4 and find the span length for which this is minimised by solving. $\partial P_{T} / \partial L=0$ to give the following expression for the amplifier span; 


$$
L_{a}=\frac{3}{\alpha}
$$

This is a remarkably simple expression which is independent of the system parameters and the majority of the fibre parameters (dispersion, nonlinearity etc). Indeed in terms of gain, this gives a universal optimum gain of $13 \mathrm{~dB}$ (or expressed linearly; $e^{3}$ ) to ensure the minimum power consumption when the system is operated at the nonlinear threshold, as given by equation 2. This is not the global minimum power which, of course, will depend on the desired SNR. But this gain is at the point where increasing the amplifier spacing not only reduces the SNR but also increases the total required system power. Thus it is desirable for both performance and power reasons not to exceed this amplifier span length which is $65 \mathrm{~km}$ for a standard loss of $0.2 \mathrm{~dB} / \mathrm{km}$

We will turn our attention to the more global case and optimise equation 3 with respect to amplifier gain. As will become clear later the minimum required total signal power is obtained through the solution of $\partial P_{T} / \partial L=0$ which may be shown to correspond to the solution of:

$$
2-e^{\alpha L}(\alpha L-2)+\frac{\sigma_{A S E} P_{T}^{3}}{\sigma_{N L} N L} \frac{e^{-\alpha L} L^{3}\left(\alpha L+e^{\alpha L}-1\right)}{\alpha}=0
$$

Equation 6 has a very simple asymptotic solution when we take the limit towards zero power (and of course zero SNR).

$$
L_{b}=\frac{2-W\left(-2 / e^{2}\right)}{\alpha}
$$

where $\mathrm{W}($.$) represents the Lambert \mathrm{W}$ function. For span lengths below $L_{b}$ the minimum required total power only increases. There is an obvious similarity between equations 5 and 7 in that both indicate a universal optimum span length only dependent on loss, and in consequence a universal amplifier gain. Ignoring the correction factor $W($.$) the difference in optimum linear$ gains is simply e, and including it the difference increases to $6 \mathrm{~dB}$. Note that in practice, the impact of nonlinearity is to reduce $L_{b}$ slightly.

\section{Exemplar graphical results}

In order to fully illustrate the implications of total power optimisation, consider an example system with the following system and fibre parameters unless otherwise stated: dispersion $20 \mathrm{ps} / \mathrm{nm} / \mathrm{km}$, loss $0.046 \quad / \mathrm{km} \quad(0.2 \mathrm{~dB} / \mathrm{km})$, nonlinearity $1.4 / \mathrm{W} / \mathrm{km}$, system length $3000 \mathrm{~km}$.

We will now plot equations 3 and results 4 to 6 on a single graph shown in figure 1 . This figure contains the essence of the results of this work and we believe rewards detailed study.
The figure shows contours of nonlinear Shannon capacity for a polarisation multiplexed system (2 $\log _{2}\left(1+S N R_{T}\right)$ ) in the amplifier spacing, total power space typically used by system designers (also shown is the amplifier gain which is useful for the fibre independent interpretation). The blue dotted line is the optimum capacity against amplifier span runs along the SNR contour ridge and diverges as the amplifier spacing tends to zero. This shows that although the vanishingly small amplifier spacing will give the maximum performance the overall power requirement tends to infinity.

The solid red line in figure 1 is the minimum total power for a given SNR i.e. the solution to equation 6 . This power is always below that of the blue dotted line and intercepts the $x$ axis at $a$ spacing given by equation 7 .

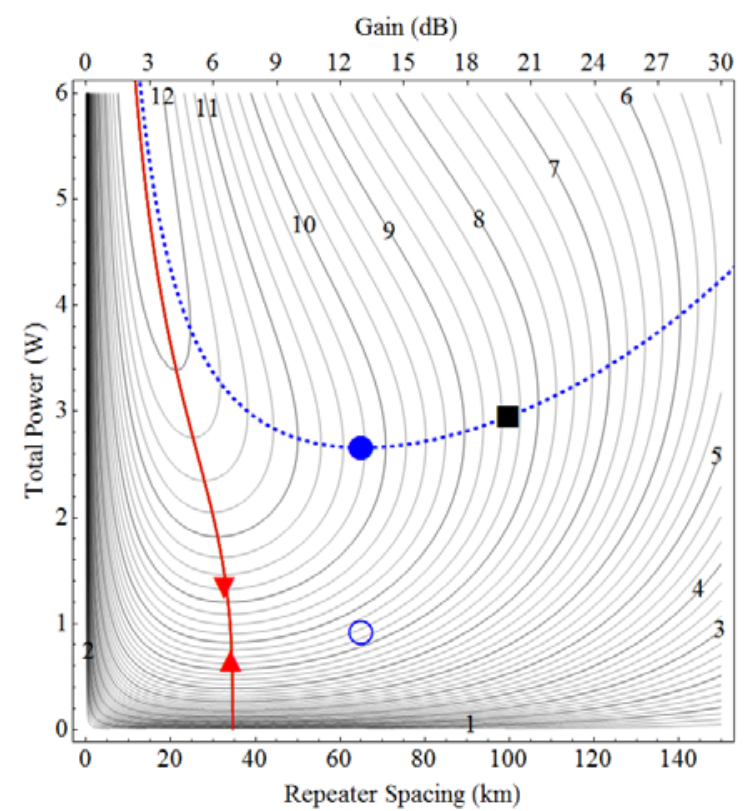

Fig 1 Total optical power $v$ amplifier spacing for the system parameters in the text. The contours are constant capacity. The blue dotted line is power giving the optimum capacity for a given amplifier span. The red line is the minimum total power for a specific capacity. Symbols represent: Solid blue circle-minimum of the optimum power curve ( always at $13 \mathrm{~dB}$ gain). Black square-optimum power at $100 \mathrm{~km}$ spacing.. Open blue circle-required power to atch capacity of black square at $13 \mathrm{~dB}$ gain. Red up and down trianglesabsolute minimum power required to achieve the same capacity as the black and blue solid symbols respectively.

A simple illustration will show how to use figure 1 and what potential energy savings are available. The black square is the optimum possible capacity with $100 \mathrm{~km}$ which is a typical terrestrial span length (with a $20 \mathrm{~dB}$ loss). The open blue circle is the point with the same capacity, but at the spacing given by equation 4 . Visually there is a substantial reduction in power 
whilst retaining the same performance. In fact the total power is reduced in that case by more than 50\% (actually 69\%). Thus the desired capacity could have been achieved with less than half the power if the amplifier spacing had been reduced from 100 to $65 \mathrm{~km}$. A reduction is almost as large (46\%) going from 80 to $65 \mathrm{~km}$ can also been seen. For the $100 \mathrm{~km}$ case a further power reduction of $-28 \%$ can be achieved by continuing on the contour to intercept the red line which for the optimum value to match the $100 \mathrm{~km}$ capacity $(34.5 \mathrm{~km})$ is almost indistinguishable from the asymptotic value in equation 7 i.e. $34.6 \mathrm{~km}$.

The second example illustrated in figure 1 shows the power saving from reducing the amplifier spacing from $L_{a}$ to $L_{b}$ whilst achieving the optimum capacity for the $65 \mathrm{~km}$ spaced system (49\%).

Figure 2 plots the power savings achievable by operating at $L_{a}$ and $L_{b}$, using the $100 \mathrm{~km}$ spacing as a reference, as a function of fibre loss. This figure shows that reducing the fibre loss monotonically reduces the benefit of operating at the optimum lengths $\left(L_{a}\right.$ and $\left.L_{b}\right)$. However, since the optimum gains are universal, energy savings are still possible by operating at either optimum length even for the lowest loss pure silica core fibre [4] and the overall optimum remains beneficial even at the theoretical loss minima of hollow core band-gap fibres [5]. With the exception of loss and amplifier spacing, we observed that the power savings were independent of all other system and fibre parameters.

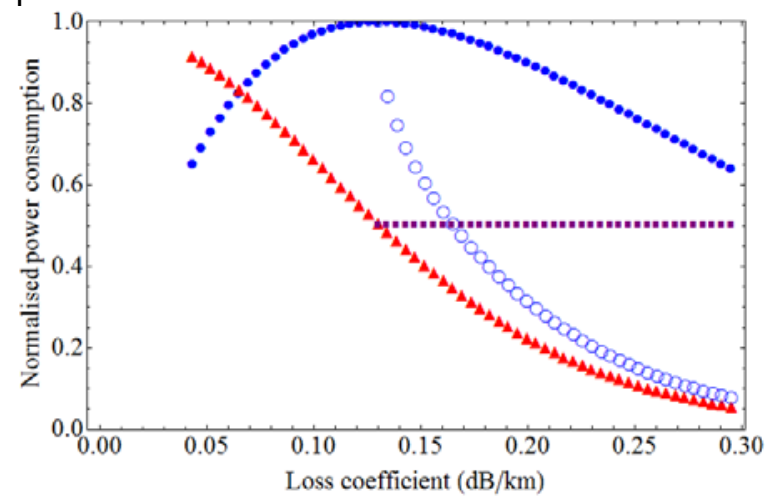

Fig. 2: Total power consumption normalized to 1 for $100 \mathrm{~km}$ (red and blue sysmols) for minimum power operation at $L_{a}$ (open blue circles) and $L_{b}$ (red triangles) and with optimized power at $L_{a}$ (open blue circles).. Also showing powe consumption ratio between optimized power at $L_{a}$ and the same capacity at $L_{b}$ (small purple squares).

Figure 3 shows the ideal amplifier spacing as defined by equation 5 (blue) or equation 6/7 (red) as a function of fibre loss coefficient. This shows the length $100 \mathrm{~km}$ is crossed by the blue line only for loss $\sim 0.13 \mathrm{~dB} / \mathrm{km}$. However a loss of $0.165 \mathrm{~dB} / \mathrm{km}$ gives a rather useful amplifier spacing of $80 \mathrm{~km}$ for the blue line. The minimum power would still favour considerably lower amplifier spacing. Fig 3 also shows the minor deviation from the asymptotic value of equation 7 achieved in practice by plotting the exact solution to equation 6 (thick red line) and the asymptotic approximation (thin red line)

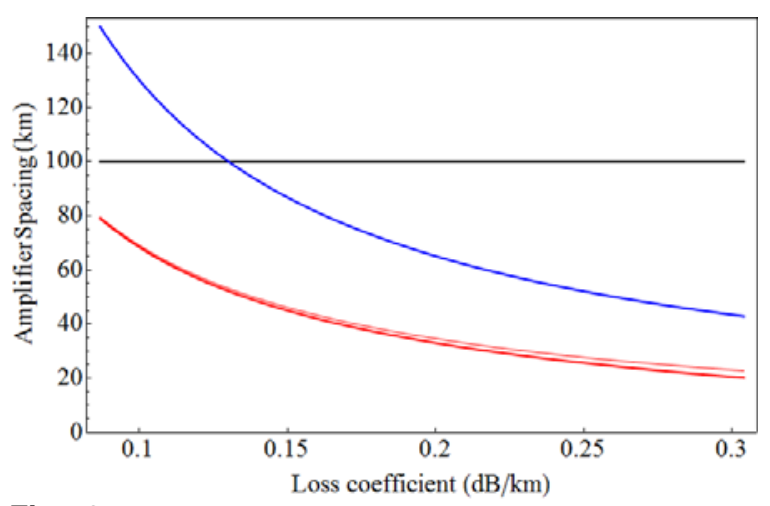

Fig. 3: The optimum amplifier spacing as defined by equation 5 (blue line) equation 6 (thick red line) and equation 7 (thin red line) against loss coefficient

\section{Conclusions}

The simple model presented here produces some very clear and widely applicable results on the power minimization of amplified fibre links. In particular we have shown that there are high potential savings (>50\%) in energy demands for optically amplified links which can be obtained by the appropriate selection the amplifier spacing (and associated output power). This spacing is generally significantly less than the current designs. We observe that a span length requiring a $13 \mathrm{~dB}$ gain will give, universally, most of the benefit. But even shorter amplifier spans will give further benefit.

Of course the savings identified here are for power or equivalently operational costs. Increased amplifier count would contribute to the capital costs. But if all systems focused on the optimum gain of $13 \mathrm{~dB}$ allowing manufacturers concentrate on a single widely deployed design then the power consumption of the world's systems would be reduced by $\sim 50 \%$ and the capital cost increase may not be so significant.

\section{Acknowledgements}

This work was partly funded by the European Communities 7th Framework Programme FP/2007-2013 grant 258033 (MODE-GAP) and the EPSRC funded project UNLOC (grant number EP/J017582/1).

\section{References}

[1] X.Chen, et al., Opt.Exp, 18, pp19039 (2010).

[2] P.Poggiolini, OFC 2013, OTh3G1 (2013)

[3] S. Gringeri et al.,Comm. Mag., 50(2), (2012).

[4] M.Hirano et al., OFC 2013, PDP5A7 (2013),

[5] N. MacSuibhne, etal.,ECOC'12,Th3A3 (2012) 